\title{
Gene Expression on Developmental Process of Porcine Pulp Cell
}

\author{
Hiroyasu Yamaguchi' ${ }^{1)}$ Takatoshi Nagano ${ }^{2)}$, Shinichiro Oida ${ }^{3)}$ and Takashi Arai ${ }^{2)}$ \\ ${ }^{1)}$ Department of General Dentistry and Clinical Education Tsurumi University School of Dental Medicine.2-1-3 Tsurumi,Tsurumi- \\ ku, Yokohama230-8501,Japan. \\ ${ }^{2}$ Department of Periodontics and Endodontics, Tsurumi University School of Dental Medicine.2-1-3 Tsurumi,Tsurumi- \\ ku, Yokohama230-8501,Japan. \\ 3) Department of Biochemistry. Tsurumi University School of Dental Medicine. 2-1-3 Tsurumi, Tsurumi-ku, Yokohama230- \\ 8501 ,Japan.
}

(Accepted for publication, December 5, 2009)

\begin{abstract}
The purpose of the current research is to elucidate of gene expression of the tooth germ. Fresh permanent incisor and molar teeth germs were extracted from mandibles of 6-month-old pig. Extracted human premolars for orthodontic reasons were used as sample of the completed tooth root. Apical and central samples were prepared from pulp tissue. mRNA expression by RT-PCR and protein expression of BMP in the pulp tissue were detected.

DMP1, BMP2 expressions were similar in all samples. OP expression was weaker in centrum of the molar tooth as compared to the other parts. OC was not expressed. BMP4 expression was strong at the incisor apical part, though it was not expressed at the molar central area. BMP5 was weakly expressed at the incisor central and molar apical areas. Though BMP7 expression was remarkably detected at the incisor and molar apexes, its expression was almost absent at the central area. Moreover for examining the specific expression of BMP7 at the apex, central superficial layer corresponding to the neck of the incisor and molar teeth was prepared. The result was that BMP7 was strongly expressed at the apex than in the central superficial layer and only a little expression was detected at central superficial layer of the incisor and molar tooth. In the current research, expressions of OP, DMP, BMP 2, 4, 5, 7 were seen in the tooth germ tissue. Thus the results suggested that expression of these genes played a central role in differentiation of the pulp. BMP7 was also suggested to have a specific role in apex formation (differentiation).
\end{abstract}

Key words: Porcine pulp cell, Tooth germ, BMP's, Dentin matrix protein, Osteopontin ,Osteocalcin

\section{Introduction}

Differentiation of vertebrate animals occurs through continuous reciprocal action between epithelial and mesenchymal system cells ${ }^{1,2)}$. Recently embryologic investigations for gene expression in each stage of tooth germ using in situ hybridization in small experimental animals are performed and BMP from TGF Beta super family has been reported to play important roles in tooth morphologic and functional regulation ${ }^{3)}$. And Aberg reported that BMP2-7 is involved in morphological formation of tooth. Especially using in situ hybridization method, BMP2, 4 and 7 has been reported to express in the bell and cap stages and play an important role in formation of enamel nodule, enamel and dentine matrixes together with influence of Shh and fgf- 4 genes on epithelial and mesenchymal cells ${ }^{4-6)}$. Although calcification and reparative dentine formation occurs in the pulp tissue after tooth

Corresponding Author: Dr. Hiroyasu Yamaguchi, Department of General Dentistry and Clinical Education Tsurumi University School of Dental Medicine . 2-1-3 Tsurumi,Tsurumi-ku, Yokohama230-8501,Japan. Tel. +8145-581-1001 Fax.+81-45-573-9599 E-mail.yamaguchi-h@tsurumi-u.ac.jp eruption through senescence period, functional expression as well as calcification mechanism of the pulp cell has not been identified.

The main matrix component forming the pulp is generally same for mesenchymal tissue, and collagen, proteoglycan as a noncollagenous protein $^{7)}$, osteopontin ${ }^{8,9)}$, osteocalcin ${ }^{10)}$, dentine matrix protein $1^{11,12)}$ etc. have been known to be produced, all of these materials are also known to be included in bone, dentine and cementum. In situ hybridization method is performed for identification, localization and distribution state of these noncollagenous matrix components. And gene expression of endocrine components of the pulp cell obtained by outgrowth method has become possible through RT-PCR. However use of in situ hybridization method in small animals, unclearness of differences between decidual and permanent teeth and differences between cell culture experiments and real organic body make difficult evaluation of metabolic activity. Thus so far we used pig jawbone, which is a better model for investigating the human teeth from points of developmental and biochemical aspects of bone, tooth 


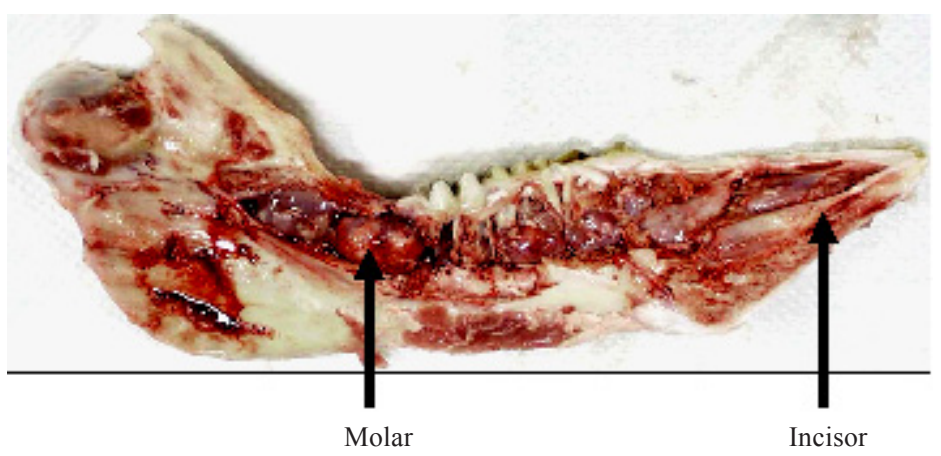

Figure 1. Extraction of tooth germ.

Molar

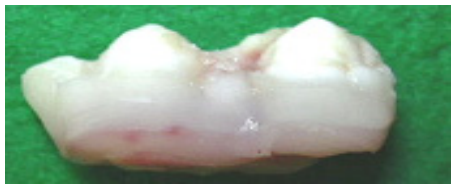

\# Apex $\bigcirc$ Central surface
Incisor

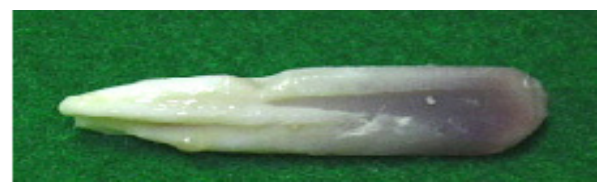

\# Apex $\bigcirc$ Central surface

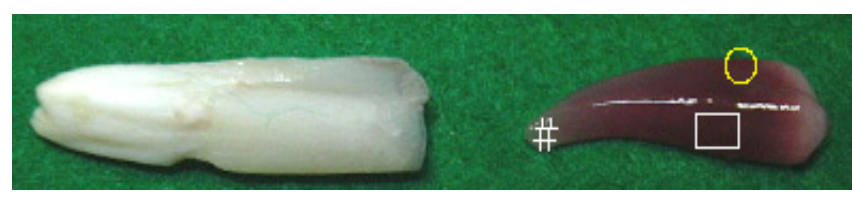

$\square$ Centrum

Figure 2. Extraction of pulp. Separation of molar tooth germ (Apex, central surface, centrum), separation of incisor tooth germ (Apex, central surface, centrum)

\section{GAPDH: S 5' ACCACAGTCCATGCCATCAC 3' AS 5' TCCACCACCCTGTTGCTGTA 3' \\ BMP-2: S 5' AAATTATGAAGCCAGCCACAG 3' AS 5' TTGACGCTTTTCTCTTTTGTG 3' \\ BMP-4: S 5' TCCTGGTAACCGAATGCTGAT 3' AS 5'GCTGCTGAGGTTGAAGACGAA3' \\ BMP-5: S 5'CTGGTATATTTGTGCCTGCTG 3' AS 5'GCATTGATGTAGCAGAAGACG3' \\ BMP-7: S 5' TCACAGCCACCAGCAACCACT 3' AS 5' GCCTGCCTCTGGTCACTGCTG 3' \\ OP S 5' TGACCTCTGTGAAAACAGCGT 3' AS 5' TGTACATTGTGAAGCTGTGAA 3' \\ DMP S 5' CAGCAGTGAGTCTCAGGAAG 3' AS 5' CAGTCATTGTCATCTTGGTCC 3'}

Figure3. Sequence of PCR primers. Specific primers were designed based on porcine and mouse mRNAa sequencces

and periodontal tissues ${ }^{13,14}$.

Then for identification of the genes, which have important roles in development of tooth germ pulp and formation process of

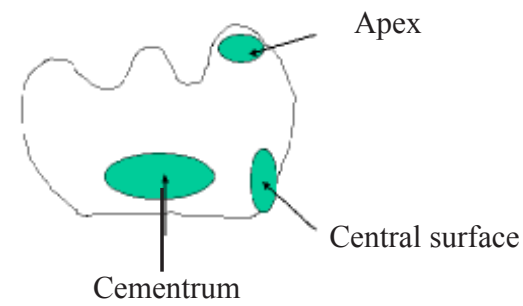

Removal of samples from part of tooth

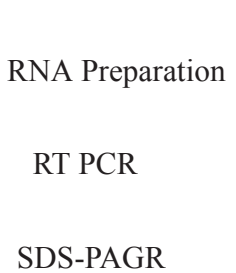

Figure 4. Experimental method. Apex, central surface, centrum. Removal of samples from each part of tooth germ. RNA extraction. RT-PCR. SDSPAGE.

dentine, expression of osteopontin (OP) as a non-collageneous protein, osteocalcin (OC), dentine matrix protein1 (DMP1), and BMP gene family as a differentiation induction factor were 


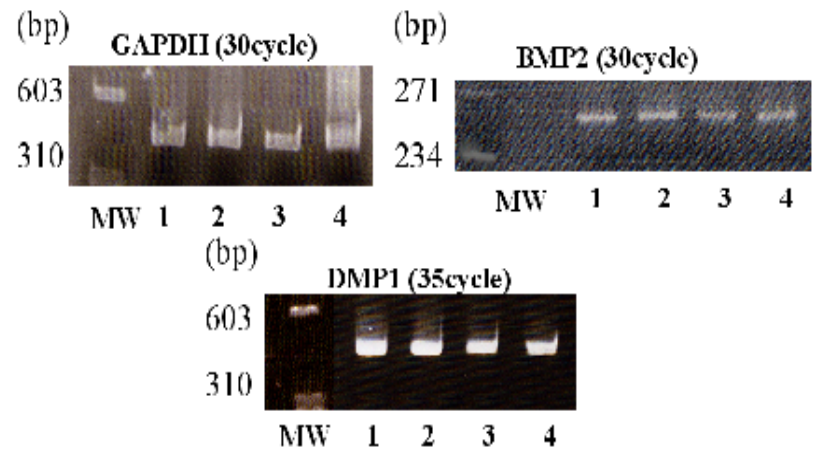

Figure 5. Genes commonly expressed in all samples. (1. Apex of permanent incisor 2. Centrum of permanent incisor 3. Apex of permanent molar 4. Centrum of permanent molar)

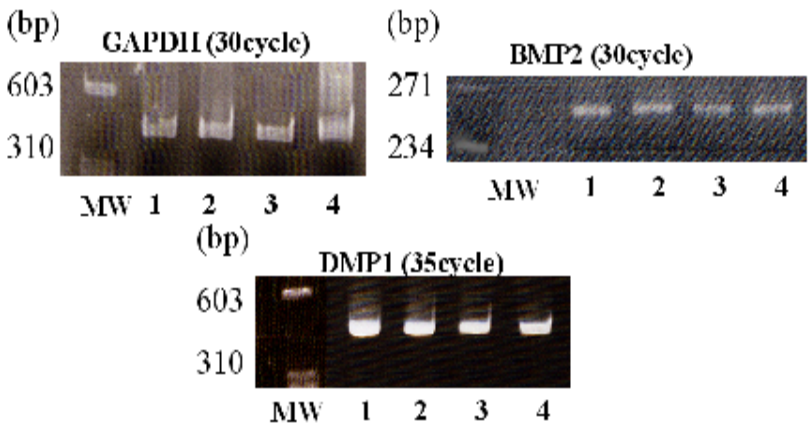

Figure 6 Genes differentially expressed in the tooth germ sections as well as each of incisor or molar teeth. (1. Apex of permanent incisor 2. Centrum of permanent incisor 3. Apex of permanent molar 4. Centrum of permanent molar)

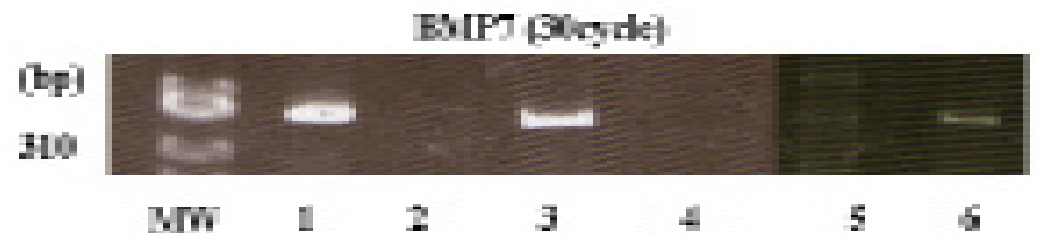

Figure 7 Genes expressed only at apex of the incisor and molar. (1. Apex of permanent incisor 2. Centrum of permanent incisor 3. Apex of permanent molar 4. Centrum of permanent molar 5. Central surface of permanent incisor 6. Central surface of permanent molar)

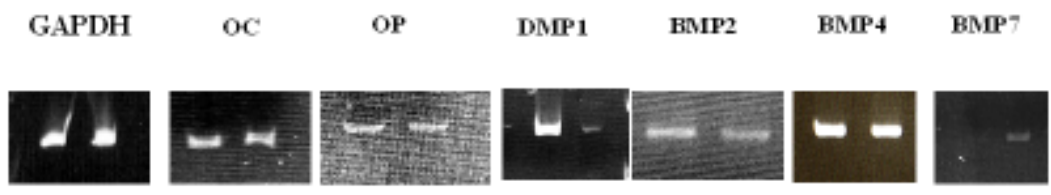

Figure8. Genes expressed in human permanent tooth. Left, apex. Right, centrum.

examined in pig tooth pulp and human premolar tooth using PCR method.

\section{Materials and Methods}

\section{1) Sampling of the tissue}

The pulp tissue removed from permanent incisor and molar teeth of 6-month-old pig mandible was used as sample after killing animal and carrying it in ice. Each pulp tissue was pulled out, and apical parts were divided and used as apical tissue (Apex:pulp horn). Then superficial part of the pulp was divided and only central part was sampled (Central surface: pulp chamber beneath the cervical margin). And to examine differences between the apical tissue and the pulp superficial surface, the pulp central part superficial layer taken as parallel to curvature of the pulp was sampled (Centrum: central pulp away from mineralized dentin). Again to compare erupted and fully developed tooth, pulp of human first premolar teeth removed for orthodontic reasons were used after obtaining the informed consent (Figures 1, 2).

\section{2) Detection of gene expression by PCR}

Total RNA from the removed pulp was prepared by Strataprep Miniprep kit (Stratagene Co. Ltd., USA) and PCR templates from total RNA were synthesized using Ready to go T-Primed First Strand Beads Kit (Pharmacia Co. Ltd., USA). Then primers were designed using the sequence information of pig and mouse (Figure 3). PCRs were performed, followed by confirmation of the products through running on a $4.5 \%$ polyacrylamide electrophoresis. Figure 4 displays the outline of the methods.

\section{Results}

\section{1) Permanent tooth germ}

DMP1 and BMP2 showed similar expressions in all samples 


\section{J.Hard Tissue Biology Vol. 19(1):17-22, 2010}

(Figure 5). Expression pattern of OP, BMP4, 5 was different in the incisor and molar teeth. OP demonstrated weak expression in the molar tooth central part as compared to the other sections. BMP4 displayed strong expression in apical area of the incisor, yet no expression in central part of the molar tooth. BMP5 was weakly expressed in central area of the incisor and apex of the molar tooth (Figure 6).

Again BMP7 showed remarkable expression in apical area of the incisor and molar tooth, yet almost no expression in central part of the teeth. Moreover for examining the specific expression of BMP7 at the apex area, central superficial surface corresponding to the neck of the incisor and molar teeth was prepared. The result was that BMP7 was strongly expressed at the apex than in the central superficial surface and only weak expression was detected at central superficial surface of the incisor and molar tooth (Figure 7). OC was not detected in any of the samples.

\section{2) Human premolar tooth}

$\mathrm{OC}$, which was not expressed in the pig permanent tooth germ, was detected both in the apex and centrum. BMP7 was not expressed in the apex but showed weak expression in the centrum. DMP1 displayed strong expression in the apex. Other gene expressions were detected both in the apex and the centrum in all samples (Figure8).

\section{Discussion}

The pulp is called as primitive connective tissue and composed of connective tissue with components of intensely calcified odontoblasts and undifferentiated mesenchymal cells. The pulp cell shows alkaline phosphatase activity, which is specifically related with calcification ${ }^{12)}$.

Non-collageneous protein osteopontin ${ }^{8,9)}$ is produced by cells of the bone, placenta, ovary, brain, tumor cell, macrophage, $\mathrm{T}$ lymphocyte etc. and promotes early differentiation of osteoblasts $^{15)}$. Ninomiya et al reported the role of osteopontin for pathological calcification in irreversible pulpitis using immunohistochemical methods ${ }^{16)}$. Osteocalcin is specifically produced in osteoblast and odontoblast, and is known to be a suppressor protein for over-calcification during bone formation ${ }^{15)}$. And Owen using calvaria cell culture experiments reported that alkaline phosphatase, type 1 collagen appeared at early bone formation, while OC and OP was recognized at later stages, showing role of these proteins in various stages of osteoblast regulation. In the current research, OC was not expressed in the developing pig tooth germ, yet detected in the permanent human premolar tooth. Since the root and pulp growth was completed in the human premolar tooth, influence of $\mathrm{OC}^{15)}$ on regulation of calcification in the pulp tissue was thought. Muramatsu el al reported that $\mathrm{OC}$ expression in the pulp decreased in parallel to senescence using real-time $\mathrm{PCR}^{18)}$. And because $\mathrm{OC}$ has been reported to inhibit overcalcification ${ }^{17)}$, detailed mechanism of calcification in the pulp is also planned to investigate.

DMP $1^{10,11)}$ has strong calcium binding affinity and is known to be a calcification marker ${ }^{19)}$. In the current research, since this molecule appears in the permanent tooth germ as well as erupted decidual human premolar, it should be different than OC, and is considered to be secreted even after eruption. In future, detailed investigation regarding expression period of this gene should be thought.

In the current research, we investigated gene expression state especially apical and central regions of the incisor and molar teeth for the premolar tooth with fully completed functional root and the permanent tooth germ without function and associated with continues formation of the enamel and dentine. However, RNA extraction from erupted tooth of the identical pig jawbones was difficult due to extremely low cell concentration especially at the apical region. Thus comparison was done for the premolar tooth with functional root instead of decidual tooth in the jawbone. And using extracted RNA from human premolar tooth, expression of the gene above was confirmed. However, since genes like BMP regulates tooth morphology and are detected in the erupted tooth, other regulatory functions are also considered to exist and our next study includes detailed examination of the gene expression in the erupted tooth.

BMP has a role in regulation of morphology and differentiation of many cell types. The tooth in development of the spinal organ is regulated with continuous reciprocal signals of the epithelium and gel layers, and especially BMP2, 4, 7 in the bell and cap staged tooth germ is related with reciprocal function between the epithelium and mesoderm and is supposed to be central regulators of the tooth morphological regulation ${ }^{4}$.

In research using in situ hybridization of the tooth germ of 6month-old ABERG mouse ${ }^{1)}$ (considered in P1 period due to enamel and dentine precipitation), BMP2 and BMP7 expressions were detected at the apical region similar to results of the current research. These results show that tooth morphological regulation by BMP support results of in situ hybridization in small animals. Thus expression of the genes is planned to investigate using samples from various growth stage of tooth germ.

In the current research, prominent expressions of OP, DMP and BMP were detected in pulp tissue of 6-month-old permanent tooth germ, suggesting the important role of these genes in developmental differentiation of the pulp. Especially BMP7 prominently expressed in apical region of the incisor and the molar teeth pulp at enamel and dentine precipitation periods, suggesting their essential role in apex formation of this time. And similar results were obtained from in situ hybridization studies ${ }^{4)}$ though different animals were used, supporting the role of BMP2, 4 and 7 in development and morphological regulation of the tooth.

OC, which is not expressed at the tooth germ, was detected in 
Hiroyasu Yamaguchi et al.: Gene Expression of Porcine Pulp Cell

human premolar tooth, and since this protein is decreased with aging ${ }^{18)}$, we are planning to investigate differences of the gene expression in the pulp in various tooth species including human as well as during aging, and further functional analysis of these genes.

\section{Conclusions}

In the current research, to identify role of the gene responsible for pulp development and dentine formation process, the followings were shown by PCR analysis using pig tooth germ and human premolar tooth:

1) OP, DMP, BMP2, 4, 5, and 7 expressions were determined in tooth germ tissue, suggesting their important roles in pulp differentiation.

2) BMP7 was considered to have a specific function for apex formation (differentiation)

\section{References}

1. Grobstein C. Mechanisms of organogenetic tissue interaction Natl Cancer Inst Monogr 26:279-299, 1967

2. Gurdon JB. The generation of diversity and pattern in animal development. Cell 24:185-199,1992

3. Vainio S, Karavanova I, Jowett Aand Thesleff I. Identification of BMP-4 as a signal mediating secondary induction between epithelial and mesenchymal tissues during early tooth development. Cell 75 :45-58, 1993

4. Aberg T, Wozney J and Thesleff I. Expression patterns of bone morphogenetic proteins (Bmps) in the developing mouse tooth suggest roles in morphogenesis and cell differentiation. Dev Dyn 210:383-396, 1997

5. Jernvall J, Kettunen P, Karavanova I, Martin LB and Thesleff I. Evidence for the role of the enamel knot as a control center in mammalian tooth cusp formation: non-dividing cells express growth stimulating Fgf-4 gene. Int J Dev Biol 38:463469, 1994

6. Vaahtokari A, Aberg T, Jernvall J, Keranen S and Thesleff I. The enamel knot as a signaling center in the developing mouse tooth. Mech Dev 54:39-43, 1996

7. LindeA. A study of the dental glycosaminoglycans from permanent human teeth and rat of the incisor. Arch oral biol $18: 49-59,1973$
8. Yokota $\mathrm{M}$, Nagata T, Ishida $\mathrm{H}$ and Wakano Y. Clonal dental pulp cells(RPD4-1,RPC-C2A) synthesize and secrete ostepontin(SPP,2ar).Biochem Biophys Res Commun 189:892-898, 1992

9. Nagata T, Yokota M, Nishikawa S, Ishida H and Wakano Y. Osteopontin expression in clonal dental pulp cells. Ann N Y Acad Sci 760:342-345, 1995

10. Nishikawa H. A study of extracellular matrix protein expressions and TGF- $\beta$ effects in clonal dental pulp cells in vitro Shikoku dental research 10:81-94, 1997

11. Kasugai S,Adachi M and Ogura H. Establishment and characterizationof a clonal cell line (RPC-C2A) from dental pulp of the rat incisor. Arch Oral Biol 33:887-891,1988

12. Feng JQ, Huang H, Lu Y, Ye L, Xie Y, Tsutsui TW, Kunieda T, Castranio T, Scott G, Bonewald LB and Mishina Y. The Dentin matrix protein 1 (Dmp1) is specifically expressed in mineralized, but not soft, tissues during development. J Dent Res 82:776-80, 2003

13. Yamaguchi H. Non-collagen proteins in porcine mandible. Tsurumi university dental journal 17:87-100, 1991

14. Kobayashi K.Proteins in the cortical and spongy bone of porcine mandible. Tsurumi university dental journal 20: 5568,1994

15. Hayakawa T, Suda T and Kizaki H. Oral biochemistry 3rd ed: pp153, Ishiyaku publisher, Tokyo, 2000

16. NinomiyaM,Oishi M,Kido J,Ohsaki Y,Nagata T. Immunohistochemical localization of oteopontin in human pulp stones. J Endod 27:269-272, 2001

17. Owen TA, Aronow M, Shalhoub V, Barone LM, Wilming L, Tassinari MS, Kennedy MB, Pockwinse S, Lian JB and Stein GS. Progressive development of the rat osteoblast phenotype in vitro: reciprocal relationships in expression of genes associated with osteoblast proliferation and differentiation during formation of the bone extracellular matrix. J Cell Physiol 143: 420-430, 1990

18. Muramatsu T, Hamano H, Ogami K, Ohta K, Inoue T and Shimono M. Reduction of osteocalcin expression in aged human dental pulp. Int Endo J 38:817-821,2005

19. Almushayt A,Narayanan K, Zaki AE and GeorgeA. Dentin matrix protein 1 induces cytodifferentiation of dental pulp stem cells into odontoblasts. Gene ther 13:611-620, 2006 
J.Hard Tissue Biology Vol. 19(1):17-22, 2010 